\title{
Cancer proteomics: The state of the art
}

\author{
Paul C. Herrmann ${ }^{\mathrm{a}}$, Lance A. Liotta ${ }^{\mathrm{a}}$ and \\ Emanuel F. Petricoin III ${ }^{\mathrm{b}, *}$ \\ ${ }^{a}$ Clinical Proteomics Program, Laboratory of \\ Pathology, National Cancer Institute, National \\ Institutes of Health, Bethesda, MD 20892, USA \\ ${ }^{\mathrm{b}}$ Clinical Proteomics Program, Division of \\ Therapeutic Proteins, CBER, Food and Drug \\ Administration, Bethesda, MD 20892, USA
}

\begin{abstract}
Now that the human genome has been determined, the field of proteomics is ramping up to tackle the vast protein networks that both control and are controlled by the information encoded by the genome. The study of proteomics should yield an unparalleled understanding of cancer as well as an invaluable new target for therapeutic intervention and markers for early detection. This rapidly expanding field attempts to track the protein interactions responsible for all cellular processes. By careful analysis of these systems, a detailed understanding of the molecular causes and consequences of cancer should emerge. A brief overview of some of the cutting edge technologies employed by this rapidly expanding field is given, along with specific examples of how these technologies are employed. Soon cellular protein networks will be understood at a level that will permit a totally new paradigm of diagnosis and will allow therapy tailored to individual patients and situations.
\end{abstract}

Keywords: Proteomics, laser capture microdissection, cancer, signal transduction

\section{Introduction}

The human genome is now mapped [1,2]. For the first time in history it is possible to take the full measure of human heredity. Genomics is impacting science with seemingly endless possibilities; however, the challenges presented by cancer continue to be quite daunting. Cancer still lacks a definition based on molecu-

\footnotetext{
* Address for correspondence: Emanuel F. Petricoin III, Tissue Proteomics Unit, Division of Therapeutic Products, Center for Biologics Evaluation Research, FDA, Bldg. 29A Room 2B02, 8800 Rockville Pike, Bethesda, MD 2089, USA. E-mail: petricoin@cber. fda.gov.
}

lar criteria alone, and a completely robust correlation between cancer and DNA based changes has not been found [3]. While the genome provides the underlying blue print of life, an information archive, the proteins do the work of the cell. Most licensed therapeutics and diagnostics work by targeting or analyzing the proteome. The recent publication of the network of protein interactions in the yeast Saccharomyces cervisiae demonstrates the vast complexity buried in protein interaction networks and unrecoverable from the genome itself [4]. In the absence of an understanding of protein changes, the information contained in the genome yields only a limited view of the full repertoire of tantalizing leads for effective new drug targets and markers for early disease detection present in a cell.

The relatively new field of proteomics seeks to expand this view. Just as the genome denotes the entire DNA code of a cell, the proteome denotes the entire protein complement of a cell, quantitatively as well as qualitatively [5]. The aforementioned yeast example is illustrative of proteome complexity. A single network of 1,548 proteins encompassing 2,358 interactions was discovered in addition to several smaller networks [4]. Through concurrent evaluation of multiple variations in this proteome, a better understanding of cell function and miss-function may be gleaned.

Proteomics, from the very core, tackles a greater number of variables than does genomics. There are 20 coded amino acids rather than 4 nucleotides. Multiple copies of individual proteins exist that vary based on cellular environment as well as cell stage. To further complicate the study, no ability to amplify specific proteins outside of increasing transcription is known. The luxury of a PCR like method of amplifying a single protein molecule is nonexistent.

At first glance it might appear that simply monitoring mRNA levels would yield proteomic information. Recently, two pharmaceutical companies have entered into collaboration based on this premise [6]. Hooper et al. demonstrated an example of such a study, analyzing the effect of commensal gut flora on endothelial mRNA expression by mice utilizing cDNA methods. The authors noted that the mRNA levels of 105 transcripts changed greater than two-fold after colonization with 
B. thetaiotomicron. Seventy-one of these transcripts were assigned to known genes, while 34 transcripts were from uncharacterized genes [7]. While producing very interesting and valuable findings, this approach does not address the actual changes at the protein level. Protein concentration is not determined by mRNA level alone and translation does not occur at the same rate on all mRNA molecules present. Post-translational processing and degradation also run at variable rates [8]. In order to understand proteins and protein interaction, the peptide molecules must be studied directly.

\section{Disease markers for diagnosis and tailored treatment}

Although still in its infancy, and currently heavily focused on techniques and methods, the field of proteomics already promises many applications to clinical medicine. These fall under two main categories: the diagnosis of disease states, and the discovery of treatment targets. In the realm of disease marker discovery, major advances are occurring already, thanks to this new field. Examples include the recent documentation of potential new markers for invasive breast carcinoma, including Proliferating Cell Nuclear Antigen and some members of the stress protein family [9], a pair of markers for lung adenocarcinoma TA01/TA02 [10], and the observation by our group of a decrease in Annexin 1 in prostate cancer [11]. As the understanding of protein interaction and networks improves, markers more closely tied to disease specifics should surface. When a more complete understanding of which factors are causative in disease is gained, new targets for treatment should emerge. An early example of new target discovery is the identification of 25 signaling targets of the MAP Kinase pathway by proteomic analysis. Only five of these had been previously characterized as MKK/ERK effectors [12]. While a very early result, this report illustrates the power of proteomics in unraveling the interplay of multiple variables.

The two main categories of diagnosis and treatment targets can be brought together to specifically tailor treatment for individual patients. Tailored treatment is already being utilized in the care of Hodgkin's disease and is being considered for coronary artery disease [13, 14]. Rosenthal and Schwartz have published some criteria to be used in establishing links between genomic variations and disease in the field of patient tailored therapy. They require that the change in genetics must cause an alteration at the protein level, the beneficial and harmful phenotypes must have apparent clinical differences, the hypothesis linking genotype and phenotype must be convincing, and the number of exemplary cases must be sufficient to draw conclusions [15]. The field of proteomics is well equipped to satisfy such criteria that all require an evaluation of protein levels and changes. In the future, changes at the protein level alone without definable genomic alterations may be sufficient for individual patient tailored therapy [16, 17].

\section{Separation technology}

The first step in proteomic evaluation is choosing an appropriate specimen to study. The most easily obtained samples consist of tissue homogenates. These have the advantage of large size, but the disadvantage of heterogeneity. A bulk tumor may consist of cancerous cells, histologically diverse normal cells and stromal components - vasculature, lymphocytes, etc. [16]. The effects of disease may be diluted or masked by the non-cancerous components, and changes in the noncancerous components may be mistaken for disease markers. One method partially circumventing these problems is the production of cell line cultures, which produces a homogeneous population of cells, but may not accurately reflect the proteomic state of the original tumor in the actual patient. A recent study demonstrated only a $20 \%$ similarity in the proteomes between cell lines and laser capture microdissected tumor epithelium. In the same study, similarity between tumor and normal tissue obtained from a single patient and even between other patients was near 95\% [17].

The ideal material for evaluation should be procured in a patient matched method, isolating tumor and normal tissue from the same specimen when possible. By comparison of such patient matched material the true effects of disease can be isolated from interpersonal differences. When tissue separation is carried out on a microscopic scale, diseased cells can be specifically selected and then compared with specifically selected non-diseased cells from the same individual organ. Such a method of tissue separation has been made possible by the invention of the Laser Capture Microscope (LCM) (Fig. 1) [18,19]. After appropriate fixation and staining of a specimen on a standard microscope slide, the slide is placed on the LCM stage and a region of interest delineated. A cap with a film of low melting temperature plastic is placed over the sample and at the push of a button an adjustable circle of 6-30 micron 


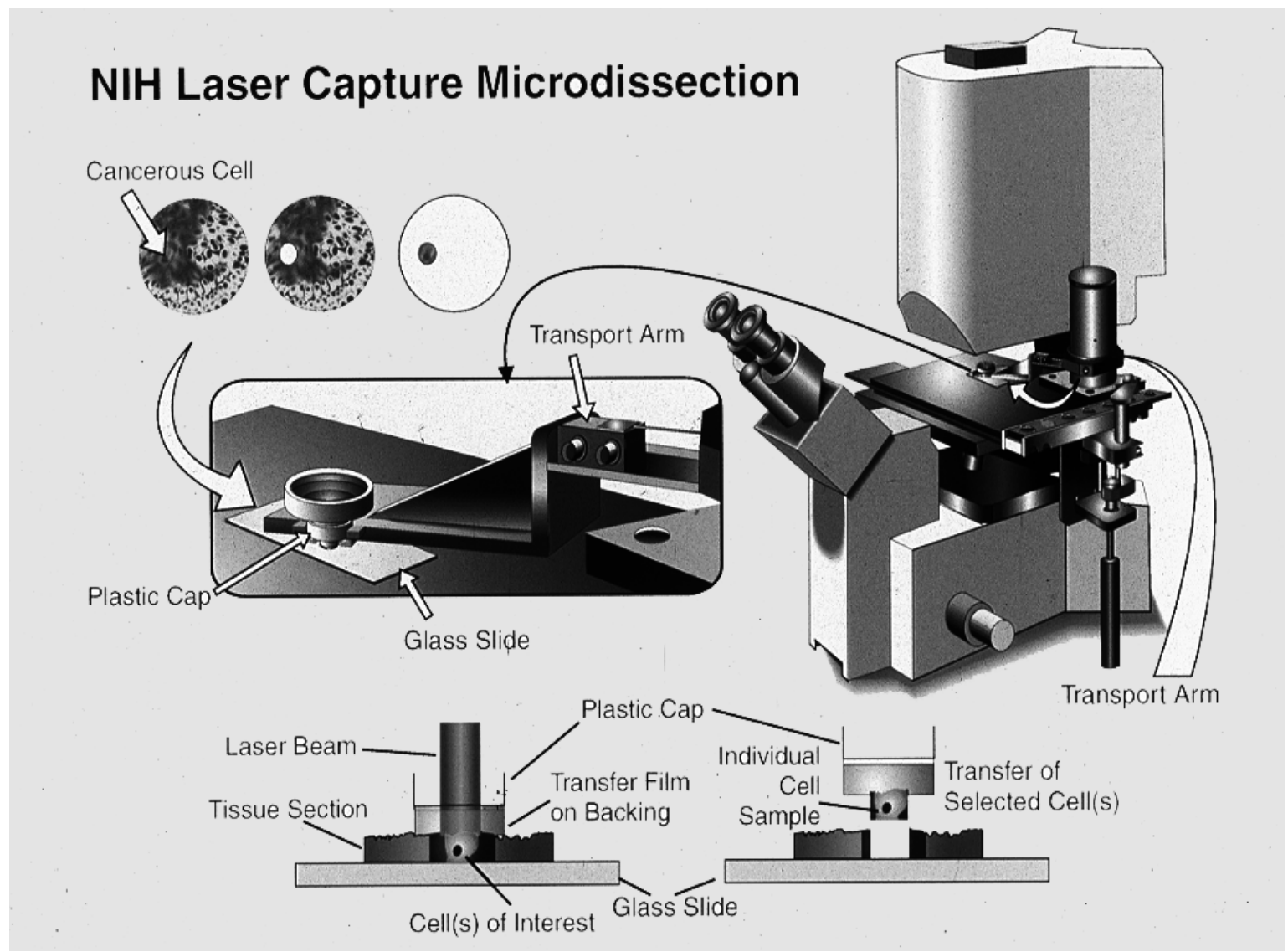

Fig. 1. Illustration of the laser capture microdissection (LCM) system. The transfer film is melted by a pulse from the laser beam and adheres to the cells of interest for transfer. Illustration is from [51].

diameter is melted onto the sample by a laser. When the cap is picked up, the tissue over which the circle was melted is adherent to the cap. The adherent cells can then be lysed by standard methods [18]. The advantages of this technique are numerous. Particularly noteworthy are those that include low energy activation that preserves the cell's original proteome unaltered, accuracy of tissue removal, and very small tissue quantities required for separation. While convenient to use, the procurement of large quantities of tissue on the order of 200,000 cells or more can be temporally prohibitive. Another microscopic tissue dissection system that has been described utilizes UV light to "blow away" tissue that is not of interest. The remaining tissue is then transferred to an appropriate medium by a laser pulse [20].

One method of tissue sampling makes use of a tissue array. Several specimens are placed adjacent to each other on a microscope slide for concurrent evaluation as illustrated by Kanonen et al. Specimens are obtained by core drilling a donor block with a thin walled sharpened stainless steel tube of $0.6 \mathrm{~mm}$ diameter. Several such core samples are then placed in an array pattern in a wax block, which is sectioned sequentially, thereby producing a polka dot tissue array on a glass slide. The tissue is then fixed and analyzed by standard immunohistochemical means [21]. The method permits the concurrent evaluation of protein expression in many specimens. However, the heterogeneity of tumor specimens dictates that occasionally samples will be excised which contain no tumor cells at all and the histologic diversity of the sample dilutes the observable effects of disease. Furthermore, analysis of protein content is limited by antigen retrieval, inherent subjectivity of immunohistochemistry and the inability to perform analysis on rare cell types such as microscopic premalignant lesions.

Recently, a new "reverse lysate array" technology by Paweletz et al. (Fig. 2) has been described which may provide a more thorough approach to translational mul- 


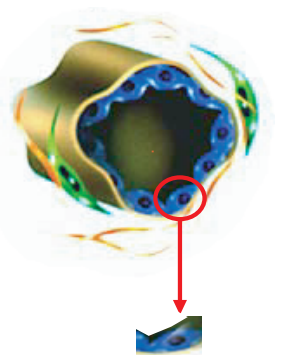

Normal Epithelium

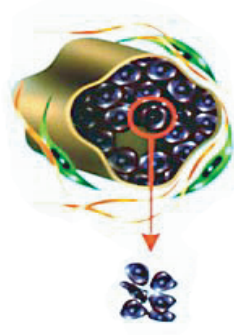

Pre-

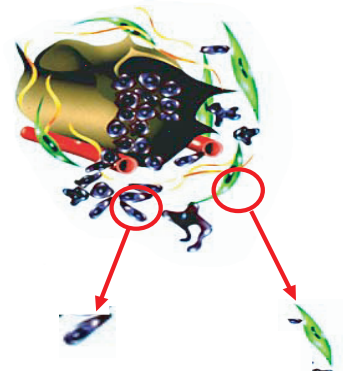

Invasive malignant
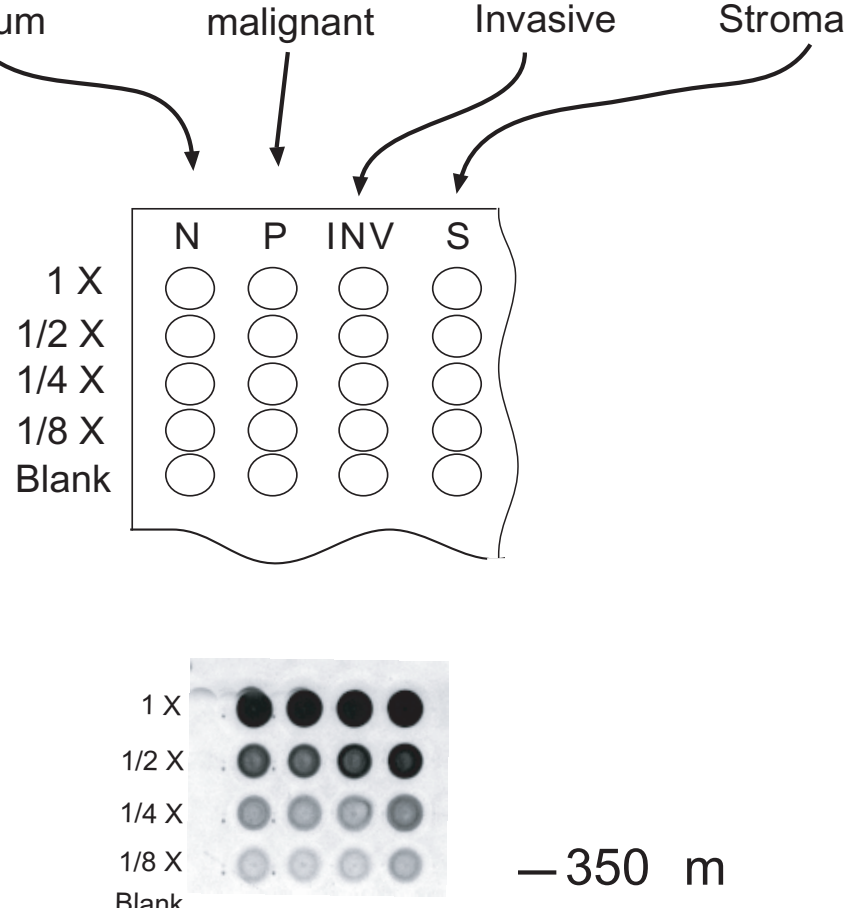

$-350 \mathrm{~m}$

Fig. 2. Illustration of protein lysate array technique. Selected microdissected cells are lysed and spotted directly onto a nitrocellulose membrane. The membrane is then probed with antibodies specific to proteins of interest. Illustration is from [22].

tiplexed analyte analysis. The authors applied lysates of microdissected esophageal material by pin array to nitrocellulose membranes. The membranes then were probed by specific antibodies for the phosphorylation status of the signal proteins AKT and ERK illustrating pro-survival pathways at the cancer invasion front. The study demonstrated very high protein sensitivity. Protein quantities found in less then $4 \times 10^{-4}$ cell equivalencies were detected. Only about 1000 molecules were necessary for detection and concentration sensitivity was demonstrated through dilution curves [22]. This technology will prove very valuable for detecting low quantities of protein. By using longitudinal patientmatched microdissected material, comparison can be made between normal, low-grade and high-grade premalignant lesions, and diseased tissue from the same patient without masking by histologic diversity. Probing the arrays with antibodies provides specific protein information and arraying of samples permits very high throughput. The technique's only limitations lie in the time required to procure samples and the requirement of antibodies previously made and purified against known proteins. However, once acquired, microdissected cell lysate libraries from as few as 2000 cells can be used to produce several hundred arrays, each of which can be probed with a specific antibody of interest recognizing a new biomarker for early disease detection, surrogate endpoints for therapeutic efficacy, or even a new therapeutic target.

In the absence of specific antibodies, protein separation is one of the most important steps in the entire process. Nonspecifically detectable proteins that are in- 
separable are unobservable. No method has been found which will separate the proteome in its entirety in a single step. Methods must be used in series to separate specific parts of the proteome for analysis. Techniques such multiplexed tandem liquid and affinity chromatography followed by MS-MS nanoESI mass spectroscopy currently require concentrations of protein which deem the minor components of the human proteome currently undetectable [23]. In the future, however, this technology may ultimately provide a non gel-based solution to proteome mining. Currently, the predominant technique for protein purification and separation in proteomics is currently two-dimensional (2D) gel electrophoresis (Fig. 3). Proteins are first separated along one axis according to charge in an isoelectric focusing step. The gel is then exposed to an electrical gradient over a perpendicular axis along which proteins migrate according to the inverse of their molecular weights [24]. Better separation is achieved than by a traditional gel based on either technique alone, but it is clear that there is not a one to one spot to protein correlation. Although new approaches such as the development of "zoom gels" to expand the separation range of the technology are increasing resolving capacity, current size limitations and insensitive staining methods place restrictions on required quantities and physical characteristics of the proteins separable by this technique [23]. 2D-gel electrophoresis will probably remain useful in magnification and resolution of specific regions of the proteome, but will always have limitations precluding high-throughput assessment of the entire proteome simultaneously on a single gel format.

In the past, many investigators have analyzed lysates from cell lines [25-28] and human tissue [29-35] by 2D-PAGE to look at tumor specific alterations in protein expression for new marker and target discovery. Image databases were developed to map proteins expressed in specific cell types and at defined stages of tumor progression [36-38]. All of these annotations are derived from cell type-enriched human tissue.

The recent ability to identify new potential disease markers from actual laser capture microdissected cells from stained human tissue specimens has enabled the analysis of protein expression in not only the affected diseased epithelium, but also the surrounding stroma, normal epithelium, and importantly the premalignant lesions [39-41]. 2D-gel profiles from the LCM procured patient-matched normal and cancer epithelium have enabled the discovery of several new potential marker candidates for prostate and esophageal cancers. Intriguingly, these proteins were not detected in stromal cells procured from the same patient tissue sections.

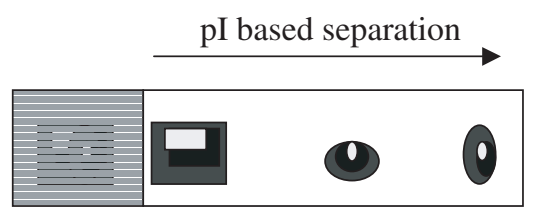

Step 1

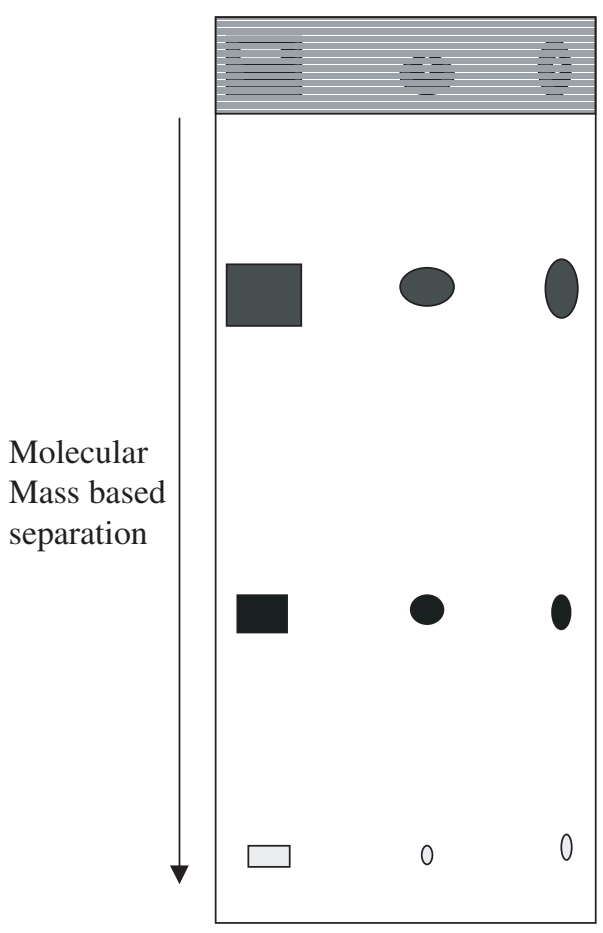

Step 2

Fig. 3. Illustration of 2-D gel electrophoresis. In step one, the proteins are separated along a narrow strip of gel on the basis of $\mathrm{pI}$ in an isoelectric focusing step. In step two, the gel strip from step one is applied to a larger gel and separation is made based on molecular mass.

\section{Protein analysis technology}

Once the proteins are separated, methods of analysis must be brought to bear on the separated entities. Protein sequencing by Edman degradation is the most specific method of analysis, but it requires a large quantity and high purity of protein [42]. Proteins removed from $2 \mathrm{D}$ gels are being sequenced by this method since the information available has not been exhausted within the current technical limitations, but other methods are needed.

Mass spectral analysis recently has been explored as a detection and protein identification method. When coupled to separation techniques, the resulting tech- 


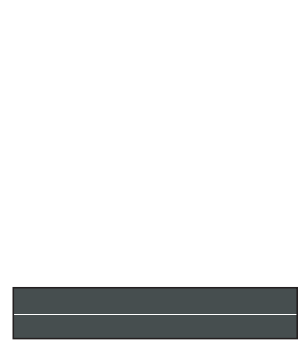

Particle Ionization

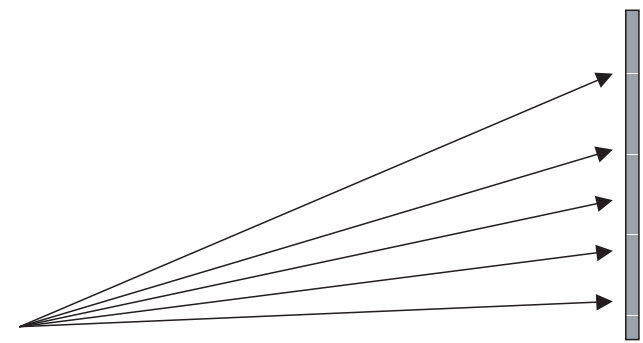

Magnetic Field

Detector

Fig. 4. Conceptual illustration of mass spectrometry. An ionized particle will have a trajectory through a magnetic field dependent on its mass to charge ratio. By allowing charged particles to pass through such a field a separation is achieved which can be analyzed by a variety of detectors.

nology can be very powerful and will probably be one of the main avenues of future exploration. After appropriate molecule charging, the mass spectrometer instrument detects molecules on the basis of their mass to charge ratio (Fig. 4). In the time of flight motif, molecules are charged and accelerated through an electric field and a recording is made of how long they take to travel a specified distance and strike a detector. The longer the time, the more massive the particle relative to its charge. Mass accuracy in the range of a few parts per million are possible through recent innovations. A more sensitive method consists of monitoring the radio frequency (rf) of a circulating population of charged particles in a cyclotron. Fourier transform of the rf signal yields the individual mass to charge ratios of the members of the population. This technique has an extremely low detection limit, but instruments are currently very expensive $[43,44]$.

Mass spectral analysis can yield sequence information, though the complete sequence cannot be determined in all cases [43-45]. Pattern analysis shows which ions or fragments contain ammonia or water losing species. Ammonia can be lost from the N-terminal amino acid, lysine or arginine, and water can be lost from serine or threonine. The fragment containing the $\mathrm{N}$-terminus is identified and computer reconstruction of fragments made. Functionalization of proteins with deuterium or reactive groups with known mass such as acetyl groups are then added and the data acquired used to further specify protein sequence. The comparison of fragment fingerprints with databases of known proteins shortens the entire process considerably. Consequently, as more proteins are discovered and characterized mass spectral analysis will improve.

Another advantage of mass spectrometry is that it can be used as a separating technique allowing analysis of an inhomogeneous sample. Tandem mass spectrom- etry uses the mass spectrometer to isolate an ionized protein. The isolated ionized protein is subsequently fragmented through a second charging cycle and the resulting fragment pattern analyzed for structural information [43-45].

Particles must be charged to be observable by mass spectrometry. The charging process has a separating effect, so picking the appropriate method allows detection of variable parts of the proteome. Electrospray excitation is accomplished by putting the molecules in a solvent in which ions are generated. The specimen is sprayed into an electric field under vacuum. In the vacuum, the uncharged solvent evaporates away, gently concentrating charge onto ionizable molecules which are then analyzed [43-45]. Matrix-Assisted Laser Desorption time-of-flight (MALDI-TOF) charges particles through excitation of the matrix by a laser [43]. The matrix then transfers energy to the species contained within it. Surface Enhanced Laser Desorption Ionization Time-of-Flight (SELDI-TOF) utilizes a similar phenomenon, except it has a unique protein baiting technology coupled to it on the front end, enabling the selection and purification of classes of proteins up-front before MALDI-based analysis. Investigators have successfully coupled this technology to LCM for the ability to discover new disease marker patterns and perform molecular fingerprinting of stages of human prostate cancer as well as rapid profiling of colon, esophageal, breast, and ovarian cancer. [46]. An example of the results of these studies is shown in Fig. 5. SELDI has recently been used in a variety of applications including protein profiling in a search for soft tissue regeneration genes [47], monitoring Alzheimer's b-amyloid production [48] and analysis of the proto-oncogene TCL1 as an Akt kinase co-activator [49]. Mendrinos et al. also recently used SELDI in the discovery of urine protein biomarkers in bladder cancer patients [50]. Both 


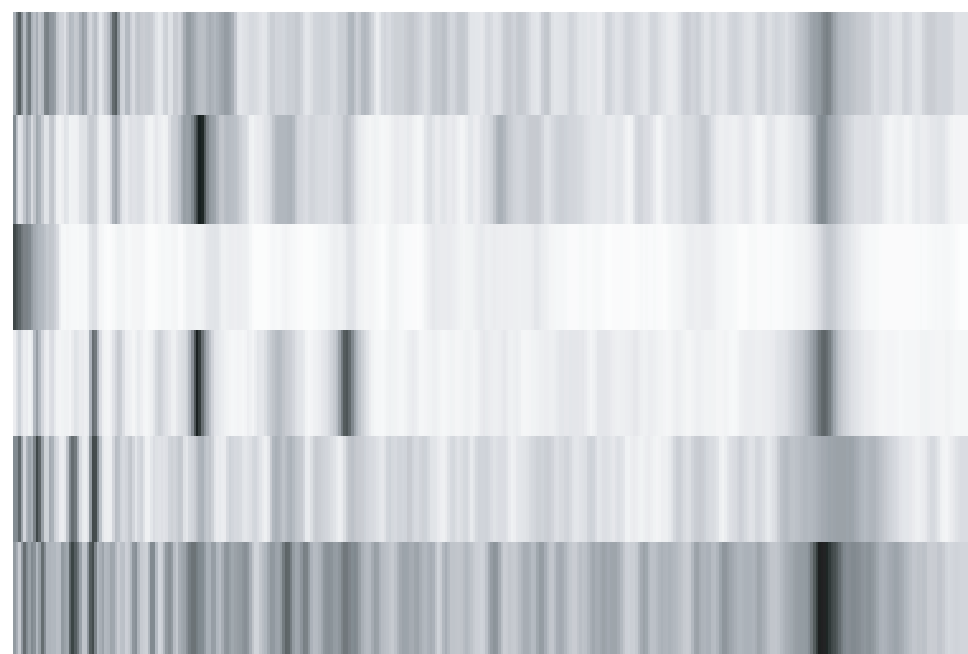

\section{Colon}

\section{Breast}

Ovary

\section{Prostate}

\section{Lung}

\section{Squamous Esophageal}

$$
\begin{aligned}
& 20000 \quad 40000 \\
& 60000
\end{aligned}
$$

Fig. 5. Illustration of molecular profiling and fingerprinting using SELDI-TOF coupled with laser capture microdissection (LCM) showing protein patterns that are unique to each human cancer type. A denstigy plot of the mass chromatogram is shown as a protein "bar code". Selected tissue is lysed and the lysate applied to a H4 reverse-phase chip. The chip is then analyzed by SELDI methodology . Illustration is from [46].

MALDI and SELDI are powerful substituents of the growing list of proteomic technologies enabling the discovery of disease markers and therapeutic targets.

\section{Looking back, looking forward}

As we look back over the last decade, many changes are apparent in the understanding of microbiology and biochemistry. The level of detail to which the various aspects of normal and aberrant cellular function, cell signaling, respiration, division, and death are understood is many times greater than it was even a few years ago. The explosion in biotechnology and the products produced for detection and treatment of disease is now only beginning in earnest. The completion of the genome project will only serve to expand the coverage and is now ushering in the next step to understanding the cellular basis of disease. Proteomics, because of its unique position for the elucidation of the components that make up the actual molecular targets for therapy and disease markers, stands poised to take up and carry on the progress made to date.

\section{References}

[1] J.C. Venter, M.D. Adams and E.W. Myers et al., The sequence of the human genome, Science 291 (2001), 1304.
[2] J.D. McPherson, M. Marra and L. Hillier et al., A physical map of the human genome, Nature $\mathbf{4 0 9}$ (2001), 934-941.

[3] A.A. Alaiya, B. Franzen, G. Auer and S. Linder, Cancer proteomics: From identification of novel markers to creation of artificial learning models for tumor classification, Electrophoresis 21 (2000), 1210-1217.

[4] B. Schwikowski, P. Uetz and S. Fields, A network of proteinprotein interactions in yeast, Nat Biotechnol 18 (2000), 12571261.

[5] S. Nock and P. Wagner, Proteomics: The post-genome revolution, Chem Unserer Zeit 34 (2000), 348-354.

[6] Press Release, Elitra Pharmaceuticals 3510 Dunhill Street, San Diego, CA 92121, July 28, 2000.

[7] L.V. Hooper, M.H. Wong, A. Thelin, L. Hansson, P.G. Falk and J.I. Gordon, Molecular analysis of commensal host-microbial relations hips in the intestine, Science 291 (2001), 881-884.

[8] S. Mullner, T. Neumann and F. Lottspeich, Proteomics - A new way for drug target discovery, Arzneimittel-Forsch $\mathbf{4 8}$ (1998), 93-95.

[9] B. Franzen, S. Linder and A.A. Alaiya et al., Analysis of polypeptide expression in benign and malignant human breast lesions, Electrophoresis 18 (1997), 582-587.

[10] T. Hirano, K. Fujioka and B. Franzen et al., Relationship between TA01 and TA02 polypeptides associated with lung adenocarcinoma and histocytological features, Brit J Cancer 75 (1997), 978-985.

[11] C.P. Paweletz, D.K. Ornstein and M.J. Roth et al., Loss of annexin 1 correlates with early onset of tumorigenesis in esophageal and prostate carcinoma, Cancer Res 60 (2000), 6293-6297.

[12] T.S. Lewis, J.B. Hunt and L.D. Aveline et al., Identification of novel MAP kinase pathway signaling targets by functional proteomics and mass spectrometry, Mol Cell 6 (2000), 13431354.

[13] G. Palmieri, A. Morabito and A. Rea et al., Tailored therapy for aggressive non-Hodgkin's lymphoma: Results of a phase II 
study with a long-term follow-up, Int J Oncol $\mathbf{1 3}$ (1998), 121127.

[14] J.W. Jukema and J.J.P. Kastelein, Tailored therapy to fit individual profiles - Genetics and coronary artery disease, Ann NY Acad Sci 902 (2000), 17-26.

[15] N. Rosenthal and R.S. Schwartz, In search of perverse polymorphisms, New Engl J Med 338 (1998), 122-124.

[16] L. Liotta and E. Petricoin, Molecular profiling of human cancer, Nat Rev Genet 1 (2000), 48-56.

[17] D.K. Ornstein, J.W. Gillespie and C.P. Paweletz et al., Proteomic analysis of laser capture microdissected human prostate cancer and in vitro prostate cell lines, Electrophoresis 21 (2000), 2235-2242.

[18] M.R. EmmertBuck, R.F. Bonner and P.D. Smith et al., Laser capture microdissection, Science 274 (1996), 998-1001.

[19] R.F. Bonner, M. Emmert-Buck and K. Cole et al., Laser capture microdissection: molecular analysis of tissue, Science 278 1481, 1483.

[20] R. Srinivasan, Ablation of polymers and biological tissue by ultraviolet-lasers, Science 234 (1986), 559-565.

[21] J. Kononen, L. Bubendorf and A. Kallioniemi et al., Tissue microarrays for high-throughput molecular profiling of tumor specimens, Nat Med 4 (1998), 844-847.

[22] C.P. Paweletz, L. Charboneau and V.E. Bichsel et al., Reverse Phase Protein Microarrays Which Capture Disease Progression Show Activation of Pro-survival Pathways at the Cancer Invasion Front, Oncogene in press.

[23] Yates Nature Biotech paper.

[24] A. Gorg, C. Obermaier and G. Boguth et al., The current state of two-dimensional electrophoresis with immobilized $\mathrm{pH}$ gradients, Electrophoresis 21 (2000), 1037-1053.

[25] Y. Katagata and S. Kondo, Keratin expression and its significance in five cultured melanoma cell lines derived from primary, recurrent and metastasized melanomas, FEBS Lett $\mathbf{4 0 7}$ (1997), 25-31.

[26] S. Prasad, V.A. Soldatenkov, G. Srinivasarao and A. Dritschilo, Identification of keratins 18, 19 and heat-shock protein 90 beta as candidate substrates of proteolysis during ionizing radiation-induced apoptosis of estrogen-receptor negative breast tumor cells, Int J Oncol 13 (1998), 757-764.

[27] S.C. Prasad, P.J. Thraves, V.A. Soldatenkov, S. Varghese and A. Dritschilo, Differential expression of stathmin during neoplastic conversion of human prostate epithelial cells is reversed by hypomethylating agent, 5-azacytidine, Int J Oncol 14 (1999), 529-534.

[28] H.F. Yam, Z.H. Wang, P.C. Or, S.W. Wang, J. Li and E.C. Chew, Effect of glucocorticoid hormone on nuclear matrix in cervical cancer cells in vitro, Anticancer Res 18 (1998), 209-216.

[29] J.E. Celis, H. Wolf and M. Ostergaard, Bladder squamous cell carcinoma biomarkers derived from proteomics [In Process Citation], Electrophoresis 21 (2000), 2115-2121.

[30] M.J. Page, B. Amess and R.R. Townsend et al., Proteomic definition of normal human luminal and myoepithelial breast cells purified from reduction mammoplasties, Proc Natl Acad Sci USA 96 (1999), 12589-12594.

[31] H. Kovarova, J. Stulik, D.F. Hochstrasser, J. Bures, B. Melichar and P. Jandik, Two-dimensional electrophoretic study of normal colon mucosa and colorectal cancer, Appl Theor Electrophor 4 (1994), 103-106.

[32] S.R. Lawson, G. Latter and D.S. Miller et al., Quantitative protein changes in metastatic versus primary epithelial ovarian carcinoma, Gynecol Oncol 41 (1991), 22-27.
[33] K. Okuzawa, B. Franzen and J. Lindholm et al., Characterization of gene expression in clinical lung cancer materials by two-dimensional polyacrylamide gel electrophoresis, Electrophoresis 15 (1994), 382-390.

[34] C. Sarto, A. Marocchi and J.C. Sanchez et al., Renal cell carcinoma and normal kidney protein expression, Electrophoresis 18 (1997), 599-604.

[35] O.S. Soldes, R.D. Kuick and T.A. Thompson 2nd et al., Differential expression of Hsp27 in normal oesophagus, Barrett's metaplasia and oesophageal adenocarcinomas, $\mathrm{Br} J$ Cancer 79 (1999), 595-603.

[36] J.E. Celis, M. Ostergaard and B. Basse et al., Loss of adipocyte-type fatty acid binding protein and other protein biomarkers is associated with progression of human bladder transitional cell carcinomas, Cancer Res 56 (1996), 4782 4790.

[37] C.S. Giometti, K. Williams and S.L. Tollaksen, A twodimensional electrophoresis database of human breast epithelial cell proteins, Electrophoresis 18 (1997), 573-581.

[38] H. Ji, G.E. Reid, R.L. Moritz, J.S. Eddes, A.W. Burgess and R.J. Simpson, A two-dimensional gel database of human colon carcinoma proteins, Electrophoresis 18 (1997), 605-613.

[39] M.R. Emmert-Buck, J.W. Gillespie and C.P. Paweletz et al., An approach to proteomic analysis of human tumors, $\mathrm{Mol}$ Carcinog 27 (2000), 158-165.

[40] R.E. Banks, M.J. Dunn and M.A. Forbes et al., The potential use of laser capture microdissection to selectively obtain distinct populations of cells for proteomic analysis - preliminary findings, Electrophoresis 20 (1999), 689-700.

[41] D.K. Ornstein, C. Englert and J.W. Gillespie et al., Characterization of intracellular prostate-specific antigen from laser capture microdissected benign and malignant prostatic epithelium, Clin Cancer Res 6 (2000), 353-356.

[42] K. Biemann, Mass-spectrometry of peptides and proteins, Апnи Rev Biochem 61 (1992), 977-1010.

[43] I.J. Amster, Fourier transform mass spectrometry, J Mass Spectrom 31 (1996), 1325-1337.

[44] D.F. Hunt, J.R. Yates and J. Shabanowitz et al., Protein sequencing by tandem mass-spectrometry, P Natl Acad Sci USA 83 (1986), 6233-6237.

[45] P. Chaurand, F. Luetzenkirchen and B. Spengler, Peptide and protein identification by matrix-assisted laser desorption ionization (MALDI) and MALDI-post-source decay time-offlight mass spectrometry, J Am Soc Mass Spectr 10 (1999), 91-103.

[46] C.P. Paweletz, J.W. Gillespie and D.K. Ornstein et al., Rapid protein display profiling of cancer progression directly from human tissue using a protein biochip, Drug Develop Res 49 (2000), 34-42.

[47] X. Li, S. Mohan and W. Gu et al., Differential protein profile in the ear-punched tissue of regeneration and non-regeneration strains of mice: a novel approach to explore the candidate genes for soft-tissue regeneration, BBA-Gen Subjects 1524 (2000), 102-109.

[48] B.M. Austen, E.R. Frears and H. Davies, The use of Seldi ProteinChip (TM) arrays to monitor production of Alzheimer's beta-amyloid in transfected cells, J Pept Sci 6 (2000), 459469.

[49] J. Laine, G. Kunstle and T. Obata et al., The protooncogene TCL1 is an Akt kinase coactivator, Mol Cell 6 (2000), 395407.

[50] S. Mendrinos, A. Vlahou and P. Kondylis et al., Protein biomarkers discovered in urine from bladder cancer patients by 
a novel protein biochip SELDI, Am J Clin Pathol 114 (2000), 631-631.

[51] N.L. Simone, C.P. Paweletz, L. Charboneau, E.F. Petricoin and
L.A. Liotta, Laser Capture Microdissection: Beyond Functional Genomics to Proteomics, Mol. Diag. 5(4) (2000), 301307. 


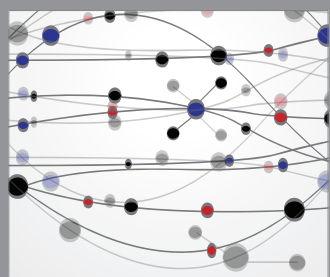

The Scientific World Journal
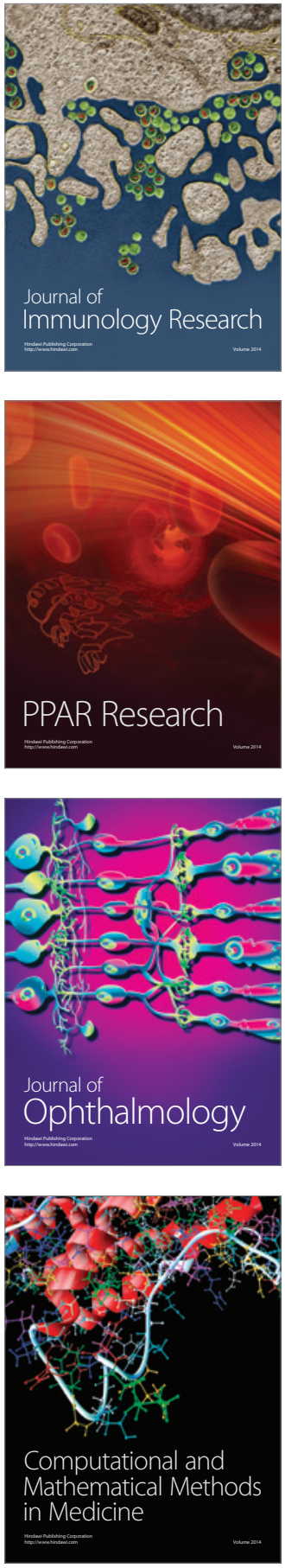

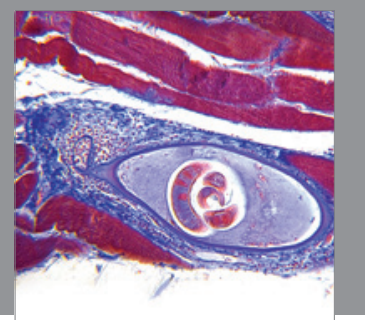

Gastroenterology

Research and Practice
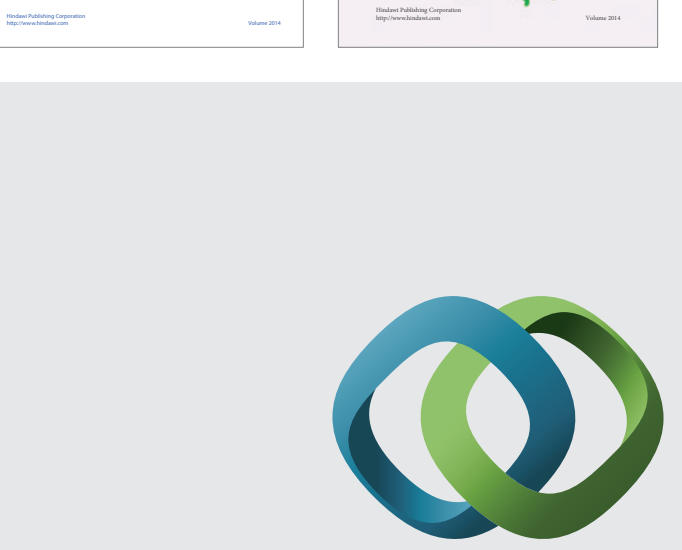

\section{Hindawi}

Submit your manuscripts at

http://www.hindawi.com
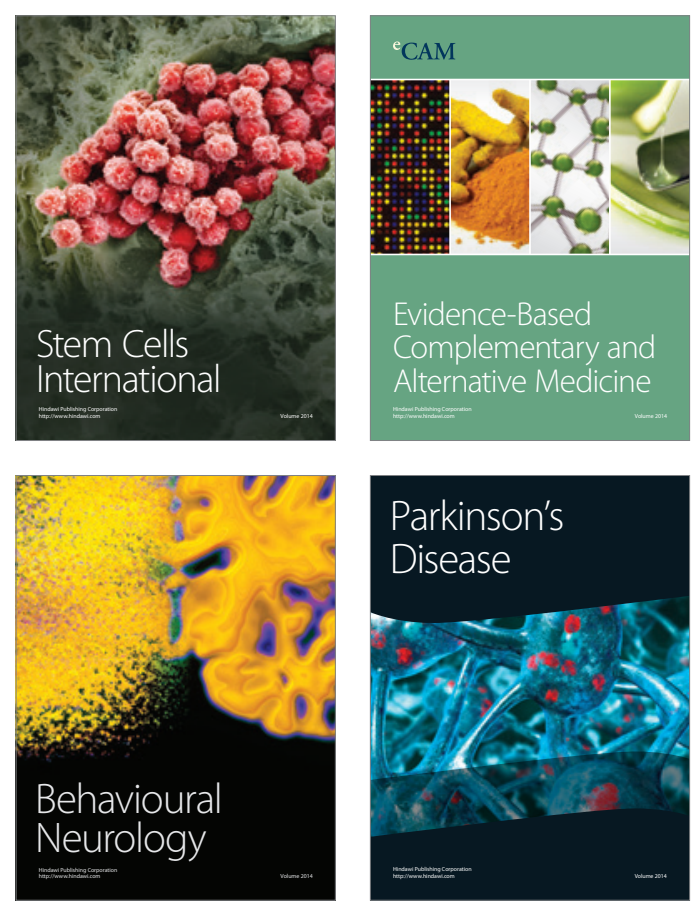

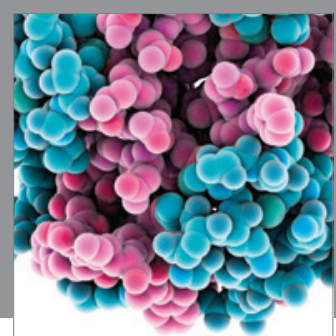

Journal of
Diabetes Research

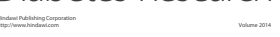

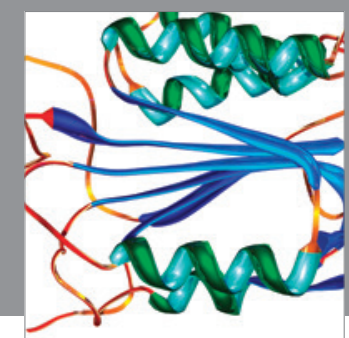

Disease Markers
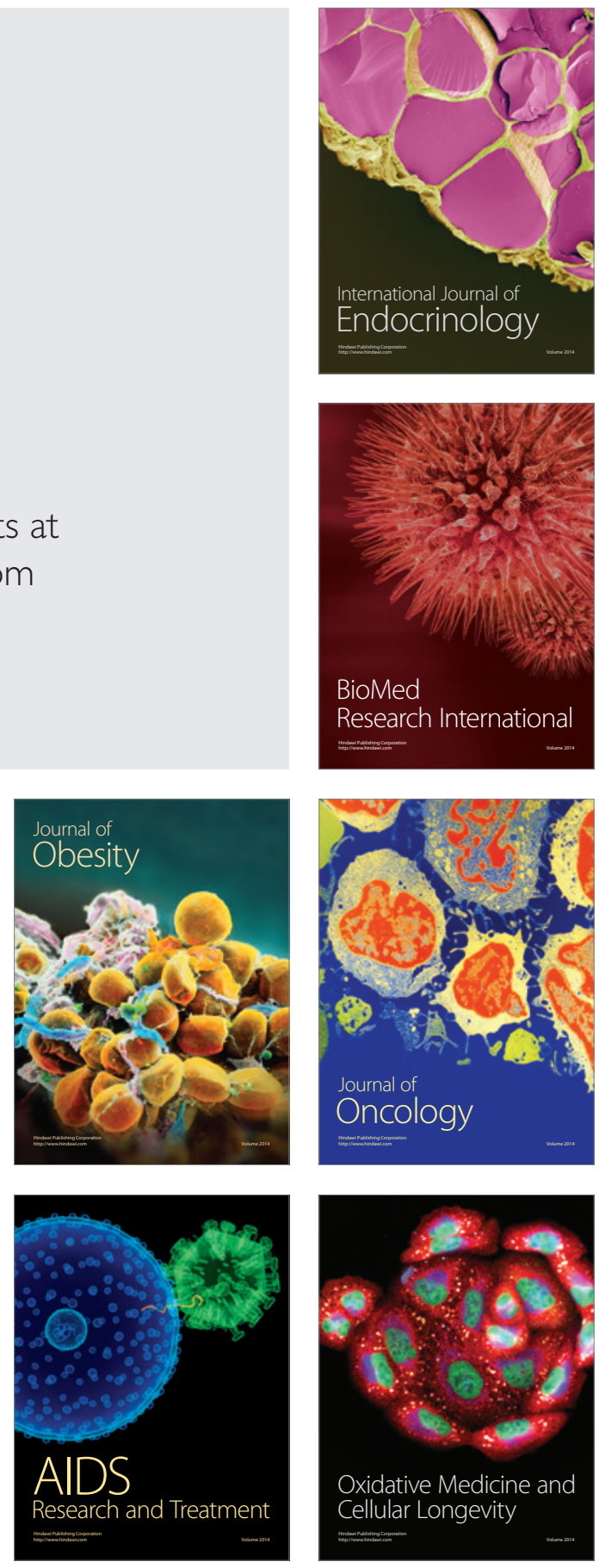